\title{
National audit of supported discharge programmes for management of acute exacerbations of chronic obstructive pulmonary disease 2008
}

\author{
Jack A Kastelik, ${ }^{1}$ Derek Lowe, ${ }^{2}$ Robert A Stone, ${ }^{2,3}$ Rhona J Buckingham, ${ }^{2}$ \\ C Michael Roberts ${ }^{2,4}$
}

'Department of Respiratory Medicine, Castle Hill Hospital, Hull and East Yorkshire NHS Trust, University of Hull and Hull York Medical School, Cottingham, East Yorkshire, UK ${ }^{2}$ Clinical Standards Department, Royal College of Physicians, London, UK

${ }^{3}$ Taunton and Somerset NHS Trust, Musgrove Park Hospital, Taunton, UK

${ }^{4}$ Barts and The London School of Medicine and Dentistry. Queen Mary University of London, London, UK

Correspondence to Dr Jack Kastelik, Department of Respiratory Medicine, Castle Hill Hospital, University of Hull and Hull York Medical School, Cottingham, East Yorkshire HU16 5JQ, UK;

jack.kastelik@hull.ac.uk

Received 30 July 2011 Accepted 15 December 2011 Published Online First 16 January 2012

\section{ABSTRACT}

The 2008 UK national chronic obstructive pulmonary disease (COPD) audit examined the use of supported discharge programmes (SDPs) in clinical practice against British Thoracic Society guidelines. 98\% of acute UK trusts participated. SDPs were available in 142 of 239 (59\%) units. 1630 of 8971 (18\%) patients with COPD were treated within SDPs. Median (IOR) stay in hospital for patients within SDPs and those not accepted for SDPs was $3(1-6)$ days and $6(3-11)$ days $(p<0.001)$, and mortality within 90 days of admission was $4.3 \%$ and $6.7 \%$, respectively. SDPs within the UK are safe and effective and reduce length of hospital stay without adverse effects on mortality.

\section{INTRODUCTION}

Over recent years alternative models to hospitalisation for managing exacerbations of chronic obstructive pulmonary disease (COPD) have been developed in the form of 'supported discharge programmes' (SDPs), which aim to avoid hospitalisation or to reduce length of hospital stay (LOS) without affecting the safety of patient care. ${ }^{1-4}$ In 2008, the Royal College of Physicians, the British Thoracic Society (BTS) and the British Lung Foundation undertook a national audit of the acute care of patients with COPD. This report reviews the data related to SDPs against audit standards described in BTS guidelines 2007. ${ }^{2}$

\section{METHODS}

All UK hospital trusts were eligible and each participating unit, defined as 'a hospital admitting acute unselected emergency admissions', completed a retrospective case note audit of up to 60 consecutive patients with admission due to COPD exacerbation identified prospectively between March and May 2008. ${ }^{5}$ The audit collected SDP data about resources and organisation of care and clinical outcomes. Also nine SDP quality indicators (table 1) were derived from BTS guidelines and units indicated whether each indicator was met in full (score 2), partially met (score 1) or not met at all (score 0 ). These scores were summed and scaled from 0 to 100 to give a SDP quality score for each unit. Similar quality scores were derived for noninvasive ventilation, pulmonary rehabilitation and long-term oxygen therapy. Data were entered by units onto a bespoke web tool and collated centrally at the Royal College of Physicians. Ethics approval was given by University College Hospital/ University College London Multicentre Research Ethics Committee (MREC). Data were analysed using SPSS V.15. Numerical data were compared using the Mann-Whitney $U$ test, and categorical data using Fisher's exact or $\chi^{2}$ test.

\section{RESULTS}

Overall 239 'units' from 180 of 184 acute NHS trusts across the UK contributed to the 2008 national COPD audit. A total of $142(59 \%)$ units reported access to SDPs. Of 9716 audit patients, $745(7.7 \%)$ died in hospital and of 8971 discharged patients (mean age (SD) 72 (10) years; forced expiratory volume in $1 \mathrm{~s} 41 \%$ (19\%) predicted), $1630(18 \%)$ were treated within SDPs. For units treating one or more audit patients within SDPs, the median LOS (IOR) was 3 (1-6) days for 1630 patients treated within SDPs, and $6(3-11)$ days for 3376 patients not accepted for SDPs $(p<0.001)$. The mortality rate at 90 days after admission was $4.3 \%$ (69 of 1591) for patients treated within SDPs and $6.7 \%$ (212 of 3172) for patients not accepted for SDPs $(p=0.001)$, with little difference in readmission rates at $34 \%$ and $35 \%$, respectively $(p=0.54)$. Units providing SDPs reported better organisation and quality than other units, in measures such as having local COPD guidelines ( $75 \%$ vs $56 \%, p=0.003$ ), discharge guidelines $(54 \%$ vs $32 \%, p=0.001)$, non-invasive ventilation quality score (lowest quartile $17 \%$ vs $33 \%, p=0.005$ ), access for all patients with COPD to respiratory nursing ( $89 \%$ vs $67 \%, p<0.001)$ and any access to formal pulmonary rehabilitation ( $94 \%$ vs $84 \%, p=0.02$ ). Units offering SDPs had lower inpatient and 90-day mortality, and lower median LOS than units without SDPs, with no significant difference in readmission rates (table 2 ).

The median (IOR) of SDP quality scores for units running an SDP was 83 (72-94). Professionals most directly involved with SDPs were respiratory nurses $(96 \%, 136$ of 142$)$, respiratory consultants $(77 \%$, 109 of 142) and physiotherapists (59\%, 84). Of 142 units providing SDPs, $16 \%$ (23) provided admission prevention, $35 \%$ (50) rapid discharge within $48 \mathrm{~h}$ of admission, 20\% (28) rapid discharge over $48 \mathrm{~h}$ from admission, and $18 \%$ (25) admission prevention and rapid discharge within and over $48 \mathrm{~h}$. There was little difference between schemes in patient 
Table 1 Quality indicators for SDPs and non-invasive ventilation Quality indicators for SDP

Quality indicators for NIV

There are clear written criteria for acceptance on to SDPs

The scheme is run by individuals who are capable of working independently and includes those specifically trained in respiratory medicine

NIV is used as the treatment of choice for persistent hypercapnic ventilatory failure during exacerbation despite medical therapy

NIV is delivered in settings that are suitable for patients with COPD; that is, a designated area where staff have been specifically trained in NIV, for example ICU, HDU, emergency admissions unit or a dedicated respiratory ward

There is a named clinician responsible for the service

There is a named consultant responsible for the NIV service

There are clear protocols of care for the patients under the SDP

There is an ongoing inter-professional training programme for all staff involved in the care of patients established on NIV

Patients accepted for SDPs are entered onto a pulmonary rehabilitation scheme, patients not accepted onto the scheme still receive a package of written smoking cessation/educational support

All patients with COPD and their carers receive written information about the SDP that describes what it is, and the support that is available well in advance of them needing the service

The SDP has good lines of communication to manage patient care together with their GP

There are clear clinical links between SDPs and various members of the primary care team

There is continuous data collection along with prospective and annual audits of the service to monitor its effectiveness

Nurses and doctors outside of specialist respiratory wards do know how to manage patients with COPD and are aware of the indications for and benefits of NIV

There is a written protocol that defines the monitoring of patients receiving NIV, and includes a minimum of regular clinical assessment, pulse oximetry and arterial blood gas measurements

There is a clear set of individualised written instructions for management of each patient receiving NIV, including what to do in the event of deterioration and agreed ceilings of therapy, along with an agreed protocol between ICU and the medical teams for escalation of NIV to ICU with invasive ventilation

Locally adapted written protocols for the management of patients with COPD requiring NIV including weaning from NIV, are available in all relevant clinical areas for all relevant staff A selection of nasal and full face masks, types and nasal pillows are available; all areas offering NIV provide written information for patients about the indications for and patient experience of NIV

There is a written policy for providing patient information about NIV to patients with severe while in a stable state, for example, in an outpatient setting or upon discharge from hospital

There is an annual audit of the use of NIV, including all clinical areas; this audit covers patients offered NIV to examine its appropriate use and those that might have benefited from NIV but who were not provided with this therapy

COPD, chronic obstructive pulmonary disease; HDU, high dependency unit; ICU, intensive care unit; NIV, non-invasive ventilation; SDP, supported discharge programme.

mortality, readmission or time spent within schemes. Units offering all three types of schemes accepted more patients for SDPs (median $39 \%$ vs $25 \%, p=0.03$ ) and had lower median LOS (median (IOR) $4(3-5)$ vs $6(5-7)$ days, $\mathrm{p}<0.001)$. Most units provided 5 -day $(65 \%, 92$ of 142$)$ or 7 -day services $(25 \%, 35$ of 142). There was little difference in patient mortality and readmission rates but 7-day services had lower LOS (median (IOR) 5 (4-6) days vs $6(5-8)$ days, $\mathrm{p}=0.004)$ and more whole time equivalent of staff involved (median (IOR) $8(5-13)$ vs 6 (4-8), $\mathrm{p}=0.009$ ).

At least 15\% (225 of 1630) of patients accepted to SDPs were outside of BTS guideline criteria, as evidenced by the presence of

Table 2 Patient characteristics and patient outcome in 142 units providing SDPs compared with 97 units not providing SDPs

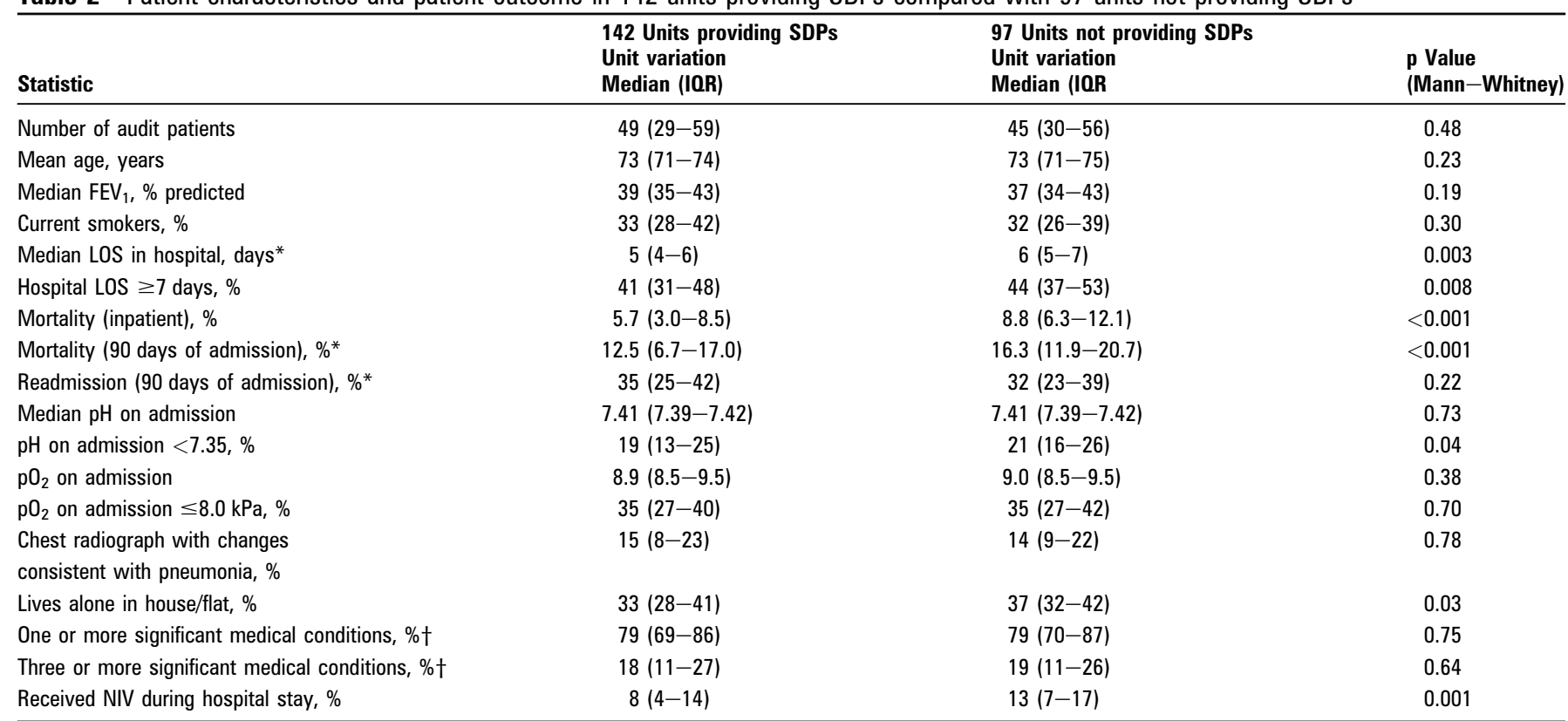

*Denotes data relating to British Thoracic Society audit criteria.

†Comorbidities included ischaemic heart disease, left ventricular failure, cor pulmonale, lung cancer or other malignant diseases, cardiac arrhythmia-for example atrial fibrillation, locomotor problems, stroke, diabetes, visual impairment, neurological conditions, alcohol-related conditions, psychiatric conditions, thromboembolic disease-for example pulmonary embolism or deep vein thrombosis, gastrointestinal conditions or endocrine disorders.

$\mathrm{FEV}_{1}$, forced expiratory volume in $1 \mathrm{~s}$; LOS, length of stay; NIV, non-invasive ventilation; $\mathrm{pO}_{2}$, partial pressure of oxygen; SDP, supported discharge programme. 
pneumonia on the chest radiograph. There was little difference in readmission rates between those with $(32 \%, 70$ of 218$)$ and those without (34\%, 466 of 1365) pneumonia; $p=0.59$ for patients within SDPs. Similarly there was little difference in readmission for patients participating in SDPs within units offering SDPs (34\% vs 35\%). There were differences in LOS for patients within SDPs with pneumonia (median (IOR) $4(2-8)$ days) and those without pneumonia $(3(1-6)$ days $)(p<0.001)$; the data were similar for patients not participating in SDPs (7 (4-12) days and $6(3-10)$ days, respectively).

\section{DISCUSSION}

This report provides information on the largest number of patients treated through SDPs from a single study. ${ }^{1-4}$ The findings from this observational audit suggest that SDPs for treating acute exacerbations of COPD are safe, reduce LOS and address queries raised by BTS guidelines. ${ }^{2}$ In the UK in 2008, $59 \%$ of units provided SDPs, a relative increase of $31 \%$ since the 2003 UK COPD audit. ${ }^{4}$ Overall 18\% (33\% in units with access to SDPs) of patients with COPD with acute exacerbations were accepted onto SDPs, supporting estimates from current BTS guidelines. ${ }^{2}$ For units treating one or more audit patients within SDPs, patients treated through SDPs had good outcomes with lower mortality at 90 days after admission than patients not participating in SDPs. Units not offering SDPs had higher inhospital and 90-day mortality, higher median LOS and more patients staying in hospital for at least 7 days (table 2). A proportion of patients treated within SDPs had abnormal chest radiographs, several comorbidities or lived alone (table 2), suggesting that with careful selection some patients can be managed within SDPs outside of the criteria described by current BTS guidelines. ${ }^{2}$

The most common type of SDP was rapid discharge within $48 \mathrm{~h}$ of admission. Units providing a combination of schemes accepted more patients for SDPs and had lower overall median LOS. Similarly, units with 7-day SDPs had shorter median LOS. Our data suggest that the optimal type of service is one that combines prevention and rapid discharge within and over $48 \mathrm{~h}$ with 7-day service provision. This requires higher staffing levels and would be of particular relevance to larger units. For smaller units a 5-day service using early discharge within $48 \mathrm{~h}$ of admission may be more appropriate. These observations support the recommendations from the current BTS guidelines. ${ }^{2}$

In conclusion, this study reported increased access and provision of SDPs in the UK and considers SDPs as safe, accepted and potentially cost effective through reduction of LOS. In the UK, almost a fifth of patients with COPD with acute exacerbations that required hospitalisation were treated within SDPs and this could be raised to at least one-third if currently applied criteria were extended to the whole of the UK. Our data revealed that more extensive SDP programmes treated more patients and had lower LOS which commissioners may find appealing. More importantly, as there were a number of positive organisational features that correlated with having SDPs, such as local guidelines, respiratory specialist nursing and access to pulmonary rehabilitation, commissioners should engage with the units who do not currently have SDPs in discussions to institute this safe and increasingly well established service.

Acknowledgements We are grateful to all the respiratory clinicians and audit teams who participated in the programme.

Funding This study was supported by the British Lung Foundation, British Thoracic Society, Royal College of Physicians and guided by the National COPD Resources and Outcomes Project (NCROP) steering group. It was funded by the Health Foundation.

\section{Competing interests None.}

Ethics approval Ethics approval was provided by University College Hospital/University College London Multicentre Research Ethics Committee (MREC).

Contributors We confirm that all the authors contributed to the manuscript.

Provenance and peer review Not commissioned; externally peer reviewed.

\section{REFERENCES}

1. Ram FS, Wiedzicha JA, Wright J, et al. Hospital at home for patients with acute exacerbations of chronic obstructive pulmonary disease: systemic review of evidence. Cochrane Database Syst Rev 2003;(4):CD003573.

2. British Thoracic Society Guidelines Development Group. Intermediate care Hospital at Home in chronic obstructive pulmonary disease; British Thoracic Society guideline. Thorax 2007:62:200-10.

3. $\mathbf{0 j o o}$ JC, Moon T, McGlone S, et al. Patients' and carers' preferences in two models of care for acute exacerbations of COPD: results of a randomised controlled trial. Thorax 2002;57:167-9.

4. Quantrill JS, Lowe D, Hosker HS, et al. Survey of early discharge schemes from the 2003 UK National COPD Audit. Respir Med 2007;101:1026-31.

5. http://www.rcplondon.ac.uk/resources/chronic-obstructive-pulmonary-disease-audit (accessed 24 Dec 2011).

Thorax Online Archive

Visit our Online Archive - available back to 1946. Subscribers may access the entire archive freely. Non-subscribers have free access to all articles prior to 2006. A simple one-time registration is required that grants access to all the free archive content, across all of our specialist titles. To view or to register visit thorax.bmj.com. 